\title{
Energy Efficient Cooperative MAC Protocols in Wireless Sensor Networks
}

\author{
Mohd Riduan Ahmad ${ }^{1}$, Eryk Dutkiewicz ${ }^{2}$ and Xiaojing Huang 3 \\ 1 Universiti Teknikal Malaysia Melaka, ${ }^{2}$ Macquarie University, ${ }^{3}$ CSIRO ICT Centre \\ ${ }^{1}$ Malaysia, ${ }^{2,3}$ Australia
}

\section{Introduction}

Multiple sensor nodes can be used to transmit and receive cooperatively and such a configuration is known as a cooperative Multiple-Input Multiple-Output (MIMO) system. Cooperative MIMO systems have been proven to reduce both transmission energy and latency in Wireless Sensor Networks (WSNs). However, most current work in WSNs considers only the energy cost for the data transmission component and neglects the energy component responsible for establishing a cooperative mechanism. In this chapter, both transmission and circuit energies for both components are included in the performance models.

Furthermore, in previous work, all sensor nodes are assumed to be always on which could lead to a shorter lifetime due to energy wastage caused by idle listening and overhearing. Low duty cycle Medium Access Control (MAC) protocols have been proposed to tackle this challenge for non-cooperative systems. In this chapter, we propose a new Cooperative low duty cycle MAC protocol (CMAC) for two cooperative MIMO schemes: Beamforming $\left(\mathrm{CMAC}_{\mathrm{BF}}\right)$ and Spatial Multiplexing $\left(\mathrm{CMAC}_{\mathrm{SM}}\right)$. Performance of the proposed CMAC protocol is evaluated in terms of total energy consumption and packet latency for both synchronous and asynchronous scenarios. All the required energy components are taken into consideration in the system performance modelling and a periodic monitoring application model is used. The impact of the clock jitter, the check interval and the number of cooperative nodes on the total energy consumption and latency is investigated. The $\mathrm{CMAC}_{\mathrm{BF}}$ protocol with two transmit nodes is suggested as the optimal scheme when operating at the $250 \mathrm{~ms}$ check interval with the clock jitter difference below $0.6 \mathrm{~Tb}$ where $\mathrm{Tb}$ is the bit period corresponding to the system bit rate.

The rest of the chapter is organized as follows. In Section 2, we briefly describe the related work. Section 3 describes the system model considered in this chapter and explains the low duty cycle protocols that we propose for cooperative transmission. Sections 4 and 5 model the system performances and the analytical results for the two cooperative MIMO schemes (BF and SM) in terms of total energy consumption and latency are presented in Section 6. Finally, in Section 7 we conclude the chapter. 


\section{Related Work}

A practical MAC that can suit cooperative transmission is required. Also, a combination of a practical MAC protocol and an efficient MIMO scheme for cooperative transmission leads to a more energy efficient and lower latency cooperative MIMO system. A combination of a MAC protocol and a virtual SM scheme for cooperative MIMO transmission has been proposed in (Yang et al., 2007) where the combined scheme achieves significant energy efficiency and lower latency. Further study has been done in (Ahmad et al., 2008a) evaluating the MAC protocol in (Yang et al., 2007) using the other two cooperative schemes: BF and Space-Time Block Coding (STBC). The authors in (Ahmad et al., 2008a) proposed that the optimal scheme for the Cooperative always on MAC (CMACON) is the BF scheme with $M=2$. However, the MAC protocols for all the schemes considered the transceivers as always being on and the networks are perfectly synchronized. Although the transmission energy is reduced and the deep fading threat is reduced, the idle listening problem is not tackled in previous research work. Also, the imperfect synchronization due to clock jitter is not considered.

Most of the duty cycle MAC protocols are designed for non-cooperative Single-Out SingleIn (SISO) schemes. Polastre in 2004 introduces B-MAC or Berkeley MAC (Polastre et al., 2004). The protocol is a variant of Carrier Sense Multiple Access (CSMA) with a preamble sampling mechanism. The preamble sampling is improved with a selective sampling method where only energy above the noise floor is considered as useful. However B-MAC experiences a long preamble problem which leads to higher transmission and reception powers. In order to reduce the long preamble problem, X-MAC (Buettner et al., 2006) proposed the use of a series of short preamble packets with the destination address embedded in the packet. The X-MAC protocol provides more energy efficient and lower latency operation by reducing the transmission energy and period burdens, idle listening at the intended receiver and overhearing by the neighbouring nodes. One concern is that the gaps between transmissions of a series of preamble packets can be mistakenly understood by the other contending nodes as an idle channel and they would start to transmit their own preamble packets which can lead to collision. One solution is to ensure that the length of the gaps must be upper bounded by the length of the listen interval.

In the same year, SpeckMAC (Wong \& Arvind, 2006) was introduced as a variation of BMAC with the idea of redundant transmission of short packets and an embedded destination address. There are two variants: SpeckMAC-Back-off (SpeckMAC-B) and SpeckMAC-Data (SpeckMAC-D). SpeckMAC-B sends short wake-up frames with an embedded target destination address many times. The problem with this scheme is that the sender wastes its transmission power by still sending the short frames although the receiver has already received it. Meanwhile, SpeckMAC-D sends the data packet which is preceded with a short preamble many times until the packet hits the receiver.

In this chapter, we propose redundant transmission of Ready-to-Send (RTS) and Clear-toSend (CTS) packets to hit the intended receiver. The cyclic RTS-CTS transmission scheme is used also for other purposes such as collision avoidance, cooperative nodes selection and channel state information (CSI) sharing between nodes. A combination of low duty cycle MAC with cyclic RTS-CTS transmission scheme is believed to reduce further the energy consumption in cooperative MIMO transmission. In addition, an imperfect synchronisation scenario due to clock jitter differences is investigated. The major contribution of this chapter is the proposal of CMAC with embedded low duty cycle mechanism which implements 
cyclic RTS-CTS transmission scheme and acknowledgement (ACK) reply to ensure higher reliability. The CMAC is suggested to be used with two cooperative schemes: optimal BF and Spatial Multiplexing. We compare the performance of both these schemes in terms of energy consumption and latency. We also include a comparison with $\mathrm{CMAC}_{\mathrm{ON}}, \mathrm{B}-\mathrm{MAC}$ and always on SISO MAC. The impact of the jitter difference, the check interval and the number of cooperative nodes on the total energy consumption and latency are investigated.

\section{System Model}

\subsection{System Description}

The baseline system for cooperative MIMO communication with the transceivers being always on is equipped with CMACON protocol as proposed and evaluated in (Jagannathan et al., 2004). Meanwhile, the baseline system for cooperative MIMO with a periodic wake-up cycle for the transceiver is equipped with the CMAC protocol as proposed and explained in sub-section 3.2. The baseline MAC for the SISO scheme with the transceiver being always on is CSMA-CA with RTS-CTS and ACK packets transmissions. For simplicity of notation, we denote the SISO scheme with this MAC protocol as the SISO always on protocol or SISO ON protocol. Also in this chapter we consider the impact of imperfect synchronization which is caused by clock jitter alone. The detailed modelling of the impact of clock jitter is given in sub-section 3.3.

The network configurations for all the schemes considered in this work are as shown in Figures 1 and 2. The network is assumed to be distributed without any infrastructure. A new node can join or leave the network at any time because the knowledge of neighbours is not important due to the fact that the selection of cooperative nodes is done during the control packets communication. We assume that there are $M$ cooperative transmitting nodes and one receiving node. A special case for the spatial multiplexing scheme is used where the number of the cooperative receivers is assumed to be $N$. Both the source and destination nodes have $n$ neighbours in their vicinity. The distance between the cooperating nodes either at the transmitting or receiving side is assumed to be very small compared to the distance between the source node and the destination node, $d$. In the case of the cooperative BF scheme, the channel information is estimated and optimized from the CTS packet by all the $M$ nodes. As for the cooperative SM scheme, the recovered data from $N-1$ nodes is forwarded to the destination node. Both schemes utilize a Maximum Likelihood (ML) detector and use a coherent receiver.

\subsection{Protocol Description}

The proposed CMAC protocol combines the advantages of the cooperative MAC with always on radios and a low duty cycle mechanism. The basic structure of the protocol is given in Algorithm 1. A node may respond to three events for the case of the BF scheme $\left(\mathrm{CMAC}_{\mathrm{BF}}\right)$ and to four events for the case of the $\mathrm{SM}$ scheme $\left(\mathrm{CMAC}_{\mathrm{SM}}\right)$. In case a node has a data packet to send where the node is acting as the source node, the basic operations for both schemes are shown in Algorithm 2.

A node starts by sending RTS packets followed by an inter-frame spacing (IFS) for a period of the length of the check interval, $T_{i}$ after sensing the channel idle. When a CTS packet is received, the source sets a timer to wake up later (the sleep duration is $T_{i}-T_{\text {cts }}-T_{\text {transient }}$ ) in order to transmit a broadcast packet at source (BS) immediately followed by the data packet 
(DATA), to its M-1 neighbours. Transmission of BS and DATA packets occurs at low transmission power due to the very short distance, $d_{m}$ between the source and its $M-1$ neighbours. The BS packet is broadcasted by the source node to recruit its neighbours for cooperative transmitting operation and the DATA packet is the original data packet provided by the sensor device. When the sending timer expires (included in the BS packet), $M$ nodes cooperatively transmit the data packet to the destination. After cooperatively transmitting the data, the source waits for an ACK packet. If an ACK is not received, the whole process is repeated. The number of RTS and CTS packets to be transmitted is given by:

$$
R=\frac{T_{i}+T_{i f s_{-} r t s}}{T_{r t s}+T_{i f s_{-} r t s}}
$$

and

$$
C=\frac{T_{i}+T_{i f s_{-} c t s}}{T_{c t s}+T_{i f s_{-} c t s}}
$$

where $T_{r t s}, T_{c t s}, T_{i f s_{r} r t s}$, and $T_{i f s_{-} \text {cts }}$ are the duration of one RTS and CTS packet and the IFS intervals for RTS and CTS, respectively. The latter are given as:

$$
T_{i f s_{-} r t s}=T_{i f s_{-} c t s} \leq T_{\text {listen }}
$$

where the value $T_{\text {listen }}$ is given in (Polastre et al., 2004). The operation of the destination node is shown in Algorithm 3 for both schemes. On receiving the RTS packet, the destination estimates the time to wake up in order to transmit CTS packets followed by IFS for a period of the length of the check interval, $T_{i}$. The sleep duration is $T_{i}-\left(S_{e q} N_{u m} \times T_{r t s}+\left(S_{e q} N_{u m}-1\right) \times\right.$ $\left.T_{i f s_{s} r t s}\right)-T_{\text {transient. }}$. After all the CTS packets are transmitted, the destination sets the timer to wake up at $T_{B S}+T_{\text {data }}-T_{\text {transient }}$ to receive the data packet. In the case of the SM scheme, the destination broadcasts the broadcast packet BR at the receiver (BR packet is broadcasted by the destination to recruit its neighbours for cooperative receiving operation.)

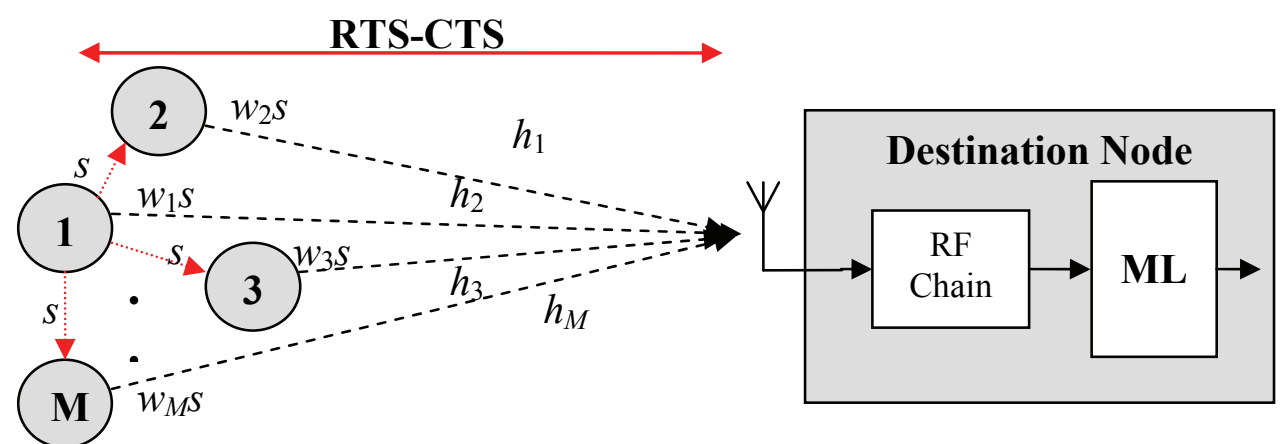

Fig. 1. A cooperative beamforming transmit diversity system with $M$ transmit nodes and destination 


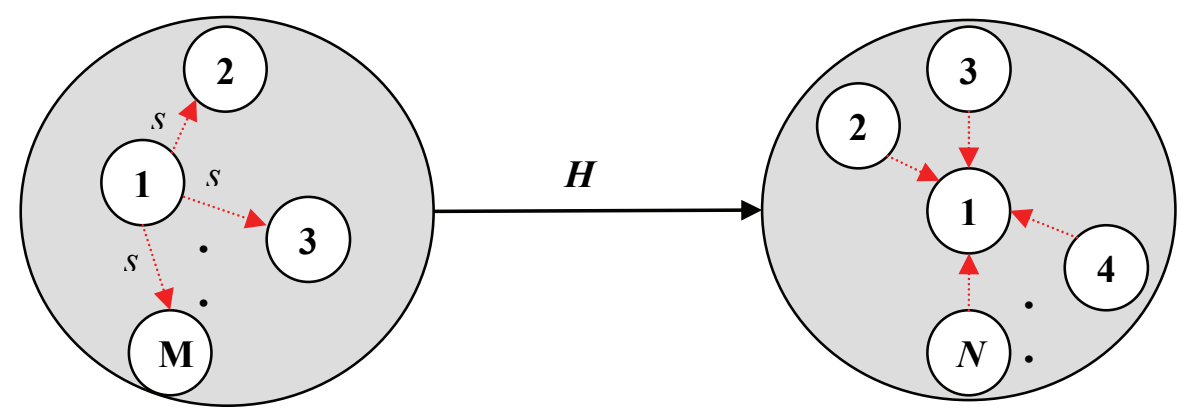

Fig. 2. A cooperative spatial multiplexing system with $M$ transmit nodes and $N$ receive nodes

first and then goes to sleep for the duration of $T_{B s}+T_{\text {data }}-T_{B r}-T_{\text {transient }}$. After receiving the data packet, the destination sends an ACK packet immediately. In the case of the SM scheme, the destination waits for its neighbours to forward the data packets and does the final decoding of the packet based on all the received copies of the data packet from its neighbours.

The operations of cooperative sending and receiving nodes are shown in Algorithm 4 and 5 . The selection of cooperative nodes is done during the control packets transmission where a node which receives RTS is informed to wake up at $T_{i}-\left(S_{e q} N_{u m} \times T_{r t s}+\left(S_{e q} N_{u m}-1\right) \times T_{i f s_{-} r t s}\right)-$ $T_{\text {transient }}$ to receive CTS. The time waiting for CTS packet is denoted as $T_{\text {wfts. }}$. If a node receives CTS, it is informed to wake up at $T_{i}-T_{c t s}-T_{\text {transient }}$ to receive BS for both schemes and BR for the SM scheme. The time waiting for the BS packet is denoted as $T_{\text {wofbsdata. }}$ The time waiting for the BR packet is the same as the time waiting for the BS packet. A node is chosen to be one of the cooperative nodes when it receives the broadcast packet. By using this mechanism, we can ensure that the network is scalable and no prior knowledge about neighbours is required for cooperative transmitting and receiving. Also, any node which does not receive CTS after receiving RTS or does not receive a broadcast packet after receiving CTS needs to go to sleep. This mechanism avoids the problems of hidden nodes. The timers' settings are described in more detail in the timing diagrams in Figures 3 and 4 for the BF and SM schemes, respectively.

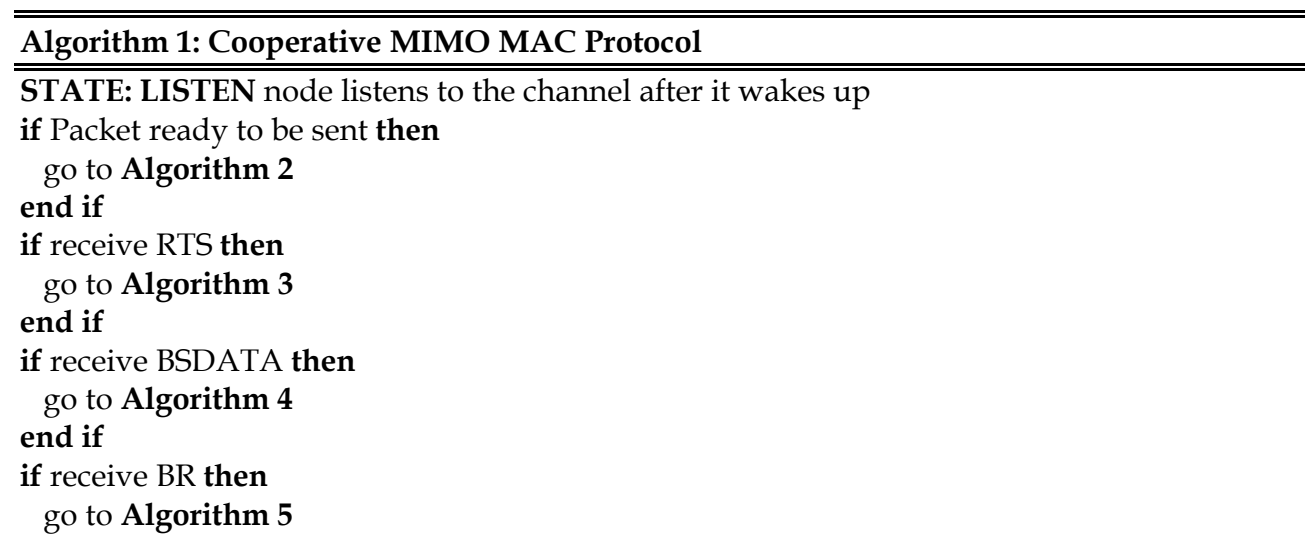


end if

Algorithm 2: Node is the source

STATE: RTS sends all RTS packets and receives CTS packet

STATE: SLEEP sets timer to wake up and goes to sleep

STATE: BSDATA broadcasts BS followed by DATA packet with low power

STATE: DATA sends data when the sending timer expires

if receive ACK packet then

go to STATE: LISTEN

else

go to STATE: RTS

end if

Algorithm 3: Node is the destination for BF scheme

STATE: LISTEN receives RTS and sets timer to wake up

go to STATE: SLEEP

STATE: CTS sends CTS packet for a period of check interval

STATE: SLEEP the node sets timer to wake up and goes to sleep

if data packet is received then

go to STATE: ACK

else if

go to STATE: LISTEN

STATE: ACK node sends ACK packet

go to STATE: LISTEN

Algorithm 3: Node is the destination for SM scheme

STATE: LISTEN receives RTS packet and sets timer to wake up go to STATE: SLEEP

STATE: CTS sends CTS packet for a period of check interval

STATE: BR sends broadcast packet to neighbours

STATE: SLEEP sets timer to wake up and goes to sleep

if data packet is received then

go to STATE: COLLECTION

else if

go to STATE: LISTEN

STATE: COLLECTION set timer to wait for data packets

if packet is not received correctly then

go to STATE: LISTEN

end if

STATE: ACK node sends ACK packet

go to STATE: LISTEN

Algorithm 4: Cooperative sending node

STATE: COOPERATIVE_SENDING nodes transmit data packet when sending timer expires

go to STATE: LISTEN listens for channel activity 

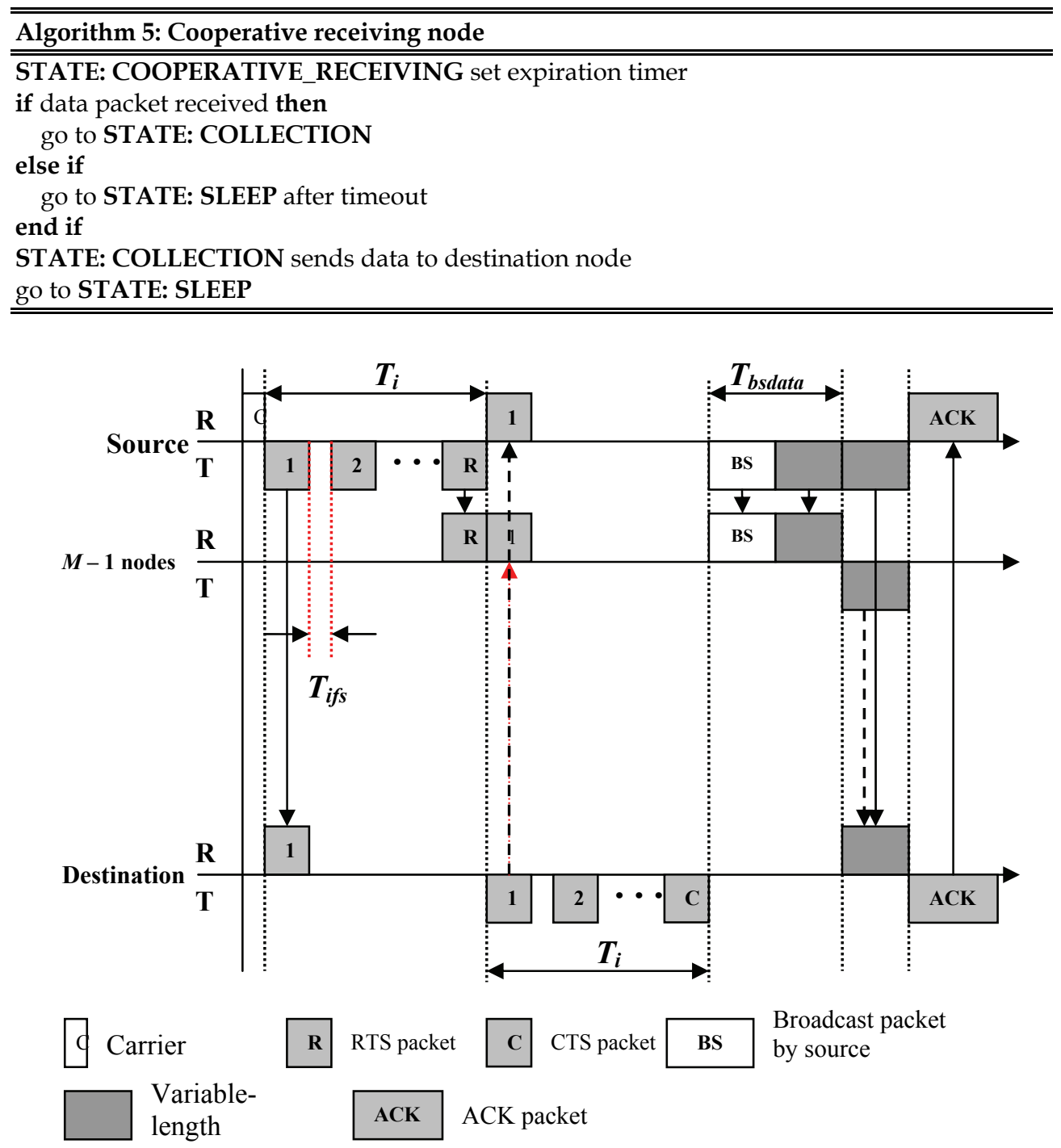

Fig. 3. Timing diagram of $\mathrm{CMAC}_{\mathrm{BF}}$ cooperative transmission 


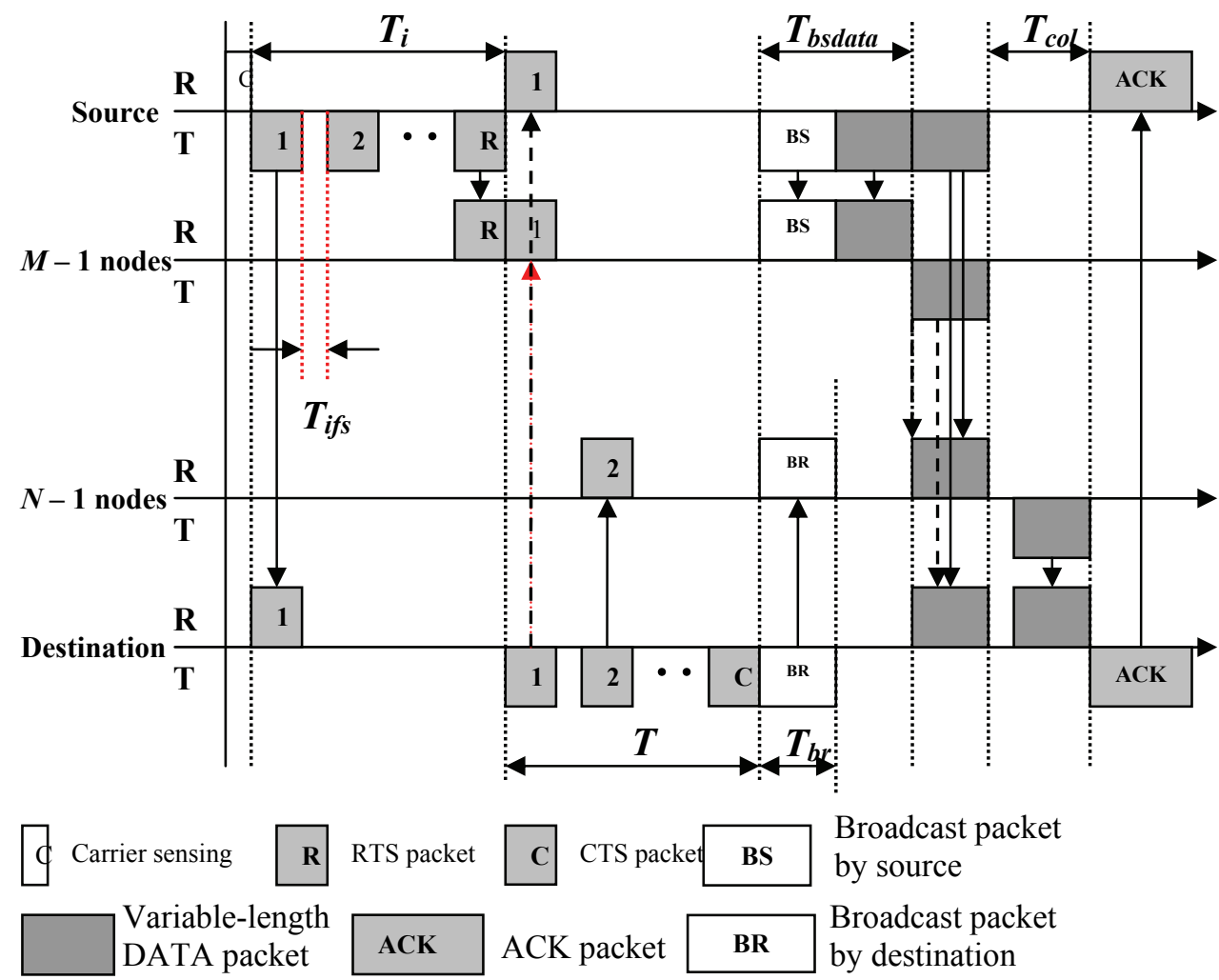

Fig. 4. Timing diagram of $\mathrm{CMAC}_{\mathrm{SM}}$ cooperative transmission

\subsection{Timing Error Model}

We consider the impact of imperfect synchronization which is caused by clock jitter alone. Each cooperative sending nodes experiences clock jitter with the jitter around a reference clock, $T_{o}$ denoted as $T_{j}^{m}$ where $1 \leq m \leq M$. The worst case scenario is considered here with only 2 cooperative transmitting nodes where the clock jitters are fixed at the extreme ends, $T_{j}{ }^{1}=-\frac{\Delta T_{b}}{2}, T_{j}{ }^{2}=+\frac{\Delta T_{b}}{2}$ where $0 \leq \Delta T_{b} \leq T_{b}$ and $T_{b}$ is the bit duration. Thus the clock jitters difference is $\Delta T_{j}=T_{j}{ }^{1}-T_{j}{ }^{2}=\Delta T_{b}$. The effect of imperfect synchronization can be modelled as a degrading function of the bit period which consequently degrades the received bit energy. Therefore the timing error as a function of the bit period and clock jitters difference is given as:

$$
T_{e}=T_{b}-\Delta T_{j}
$$




\section{Energy Consumption Performance Model}

In this section, three analytical models are developed and analyzed: $\mathrm{SISO}_{\mathrm{ON}}, \mathrm{CMAC}_{\mathrm{ON}}$ with the optimal BF scheme and $\mathrm{CMAC}$ with 2 variants, $\mathrm{CMAC}_{\mathrm{BF}}$ and $\mathrm{CMAC}_{\mathrm{SM}}$. The total energy consumption of each model is analysed and compared. The retransmission rate is modelled as a function of PER where the detailed models and analysis can be found in (Ahmad et al., 2008a).

We consider a periodic sampling application with a uniform sampling period, $T_{s}$ which has been discussed in detail (Polastre et al., 2004). In general, the energy consumed by a sensor node can be categorized into five major parts (Cui et al., 2004): energy expended during data sampling by sensor, $E_{\text {sensor, }}$ energy expended during running the transceiver circuits, $E_{c}$, energy expended during packet transmission, $E_{t}$, energy expended during packet reception, $E_{r}$ and energy expended while idle listening, $E_{i d l e}$.

For the case of the system with the CMAC protocol, additional energy must be considered: energy expended during sleeping, $E_{\text {sleep, }}$ listen energy after waking up, $E_{\text {listen }}$ and transient energy, $E_{\text {transient }}$. The cooperative mechanism establishment energy cost is included in the transmission and reception energy models. Therefore, all the energy components must be considered when comparing the total energy consumption of the cooperative MIMO and SISO transmission schemes.

\subsection{SISO System}

The total energy consumption in the SISO system, in general, is given as:

$$
E_{\text {siso }}=\left(E_{r x}+E_{c r}\right)+\left(E_{t x}+E_{c t}\right)+E_{\text {sensor }}+E_{\text {idle }}
$$

where $E_{r x}$ and $E_{t x}$ are the energy spent during reception and transmission, and $E_{c r}$ and $E_{c t}$ are the energy spent by the receiver and transmitter circuits. The transmission energy model for the SISO system which includes both the radiated power and circuit power is the same as discussed in (Ahmad et al., 2008a). Consequently, the reception energy model can be obtained directly from the transmission energy model in (Ahmad et al., 2008a). The total time a node spends during successful transmission is given as:

$$
T_{t x_{-} s}=r_{s} \times\left(N_{r t s}+N_{c t s}+N_{d a t a}+N_{a c k}\right) \times T_{t x_{-} b}
$$

and the total time a node spends during unsuccessful transmission is given as:

$$
T_{t x_{-} u}=r_{s} \times\left(N_{r t s}+N_{c t s}+N_{\text {data }}\right) \times T_{t x_{-} b}
$$

where $r_{s}$ is the sampling frequency and can be obtained by the inverse of the sampling period, $T_{t x_{-} b}$ is the transmit period per bit, and $N_{r t s}, N_{c t s}, N_{d a t a}$ and $N_{a c k}$ are the lengths of the RTS, CTS, DATA and ACK packets. The total time a node spends during successful reception is given as: 


$$
T_{r x_{-} s}=r_{s} \times\left(n \cdot N_{r t s}+n \cdot N_{c t s}+N_{d a t a}+N_{a c k}\right) \times T_{r x_{-} b}
$$

and the total time a node spends during unsuccessful reception is given as:

$$
T_{r x_{-} u}=r_{s} \times\left(n \cdot N_{r t s}+n \cdot N_{c t s}+N_{d a t a}\right) \times T_{r x_{-} b}
$$

where $T_{r x_{-} b}$ is the receive period per bit. The total time a node spends idle for successful communication is given as:

$$
T_{i d l e_{-} s}=1-T_{t x_{-} s}-T_{r x_{-} s}-T_{\text {sensor }}
$$

and the idle time for unsuccessful communication is given as:

$$
T_{\text {idle } \_u}=1-T_{t x_{-} u}-T_{r x_{-} u}
$$

where $T_{\text {sensor }}$ is the period of a sensor to start, initialise, and collect data as discussed in (Mainwaring et al., 2002; Polastre et al., 2004). Thus, the total energy consumption for successful SISO system communication can be obtained as:

$$
E_{s i s O_{-} s}=\left(P_{p a}+P_{c t}\right) \cdot T_{t x_{-} s}+\left(P_{r}+P_{c r}\right) \cdot T_{r x_{-} s}+P_{i d l e} \cdot T_{i d l e_{-} s}
$$

and the total energy consumption for unsuccessful SISO system communication can be obtained as:

$$
E_{\text {siso_u }}=\left(P_{p a}+P_{c t}\right) \cdot T_{t x_{-} u}+\left(P_{r}+P_{c r}\right) \cdot T_{r x_{-} u}+P_{i d l e} \cdot T_{i d l e_{-} u}
$$

Therefore, the total energy consumption for the SISO system can be modelled as a function of the retransmission rate:

$$
E_{\text {siso }}=\left(\frac{P_{p S I S O}}{1-P_{p S I S O}}\right) E_{\text {siso_u }}+E_{\text {siso_s } s}+E_{\text {sensor }}
$$

where $P_{p S I S O}$ is the packet error probability of the SISO system which can be obtained from (Ahmad et al., 2008a).

\subsection{Cooperative Always On MIMO System}

In this sub-section, we analyze total energy consumption for the optimal cooperative BF scheme with the $\mathrm{CMAC}_{\mathrm{ON}}$ protocol. The transmission energy model for the cooperative always on MIMO system which includes the radiated power, circuit power and cooperative mechanism power is the same as discussed in (Ahmad et al., 2008a). Consequently, the 
reception energy model can be obtained directly from the transmission energy model in (Ahmad et al., 2008a).

In order to provide better understanding about the energy models for cooperative MIMO systems in this chapter, we categorize both the transmission and reception total time into three categories which are based on packet types, namely: control, cooperative mechanism and data categories. The total time a node spends during successful control packet transmission is given as:

$$
T_{t x_{-} s_{-} \text {control }}=r_{s} \times\left(N_{r t s}+N_{c t s}+N_{a c k}\right) \times T_{t x_{-} b}
$$

and the total time a node spends during cooperative mechanism transmission for optimal $\mathrm{BF}$ scheme is given as:

$$
T_{t x_{-} \text {Bsdata }}=r_{s} \times\left(N_{B s}+N_{\text {data }}\right) \times T_{t x_{-} b}
$$

and the total time a node spends during data packet transmission is given as:

$$
T_{t x_{-} \text {data }}=r_{s} \times M \times\left(N_{\text {data }}\right) \times T_{t x_{-} b}
$$

Thus, the total time a node spends during successful transmission in cooperative always on MIMO system with optimal BF scheme can be given as:

$$
T_{t x_{-} s_{-} B F}=T_{t x_{-} s_{-} \text {control }}+T_{t x_{-} B s d a t a}+T_{t x_{-} \text {data }}
$$

and the total time a node spends during unsuccessful transmission is given as:

$$
T_{t x_{-} u_{-} B F}=T_{t x_{-} s_{-} B F}-\left(r_{s} \times N_{a c k} \times T_{t x_{-} b}\right)
$$

where $N_{B s}$ is the length of the broadcast packet at the source node. The total time a node spends during successful control packet reception is given as:

$$
T_{r x_{-} s_{-} \text {control }}=r_{s} \times\left(n \cdot N_{r t s}+n \cdot N_{c t s}+N_{a c k}\right) \times T_{r x_{-} b}
$$

and the total time a node spends during cooperative mechanism reception is given as:

$$
T_{r x_{-} \text {Bsdata }}=r_{s} \times(M-1) \times\left(N_{B s}+N_{\text {data }}\right) \times T_{r x_{-} b}
$$

and the total time a node spends during data packet reception is given as:

$$
T_{r x_{-} \text {data }}=r_{s} \times\left(N_{\text {data }}\right) \times T_{r x_{-} b}
$$


Thus, the total time a node spends during successful reception in cooperative always on MIMO system with optimal BF scheme can be given as:

$$
T_{r x_{-} s_{-} B F}=T_{r x_{-} s_{-} \text {control }}+T_{r x_{-} \text {Bsdata }}+T_{r x_{-} \text {data }}
$$

and the total time a node spends during unsuccessful reception is given as:

$$
T_{r x_{-} u_{-} B F}=T_{r x_{-} s_{-} B F}-r_{s} \times\left(N_{a c k}\right) \times T_{r x_{-} b}
$$

The total time a node spends idle for successful communication is given as:

$$
T_{\text {idle_s_B } B F}=1-T_{t x_{-} s_{-} B F}-T_{r x_{-} s_{-} B F}-T_{\text {sensor }}
$$

and the idle time for unsuccessful communication is given as:

$$
T_{i d l e_{-} u_{-} B F}=1-T_{t x_{-} u_{-} B F}-T_{r x_{-} u_{-} \_B}
$$

Thus, the total energy consumption for successful cooperative always on MIMO system communication can be obtained as:

$$
\begin{aligned}
& E_{B F_{-} s}=\left(P_{p a}+P_{c t}\right) \cdot T_{t x_{-} s_{-} \text {control }}+\left(P_{p a B s}+P_{c t}\right) \cdot T_{t x_{-} B s d a t a}+ \\
& \left(P_{p a B F}+P_{c t}\right) \cdot T_{t x_{-} \text {data }}+\left(P_{r}+P_{c r}\right) \cdot T_{r x_{-} s_{-} \text {control }}+\left(P_{r B s}+P_{c r}\right) \cdot T_{r x_{-} \text {Bsdata }}+ \\
& \left(P_{r B F}+P_{c r}\right) \cdot T_{r x_{-} \text {data }}+P_{\text {idle }} \cdot T_{\text {idle_s_B } s_{-} B F}
\end{aligned}
$$

and the total energy consumption for unsuccessful cooperative always on MIMO system communication can be obtained as:

$$
\begin{aligned}
& E_{B F_{-} u}=\left(P_{p a}+P_{c t}\right) \cdot T_{t x_{-} u_{-} \text {control }}+\left(P_{p a B s}+P_{c t}\right) \cdot T_{t x_{-} B s d a t a}+ \\
& \left(P_{p a B F}+P_{c t}\right) \cdot T_{t x_{-} \text {data }}+\left(P_{r}+P_{c r}\right) \cdot T_{r x_{-} u_{-} \text {control }}+\left(P_{r B s}+P_{c r}\right) \cdot T_{r x_{-} B s d a t a}+ \\
& \left(P_{r B F}+P_{c r}\right) \cdot T_{r x_{-} \text {data }}+P_{\text {idle }} \cdot T_{\text {idle_u_ }{ }_{-} B F}
\end{aligned}
$$

Therefore, the total energy consumption for the cooperative always on MIMO system can be modelled as a function of the retransmission rate:

$$
E_{B F_{-} o n}=\left(\frac{P_{p B F}}{1-P_{p B F}}\right) E_{B F_{-} u}+E_{B F_{-} s}+E_{\text {sensor }}
$$

where $P_{p B F}$ is the packet error probability of the cooperative BF system which can be obtained from (Ahmad et al., 2008a). 


\subsection{Cooperative Low Duty Cycle MIMO System}

In this sub-section, we analyze the total energy consumption for the cooperative BF and SM schemes equipped with the proposed cooperative low duty cycle MAC protocol. The only modifications on the total energy consumption model are the definition of the control packets intervals which should be depended on the length of the check interval where the $R$ and $C$ terms are included and the addition of sleep energy. Also, the idle listening cost still exists when a node is in listening and waiting states. The transient energy is included in the total listening energy cost as explained in details in (Polastre et al., 2004). The total time a node spends during successful control packet transmission in cooperative low duty cycle MIMO system is given as:

$$
T_{t x_{-} s_{-} \text {control }}=r_{s} \times\left(R \cdot N_{r t s}+C \cdot N_{c t s}+N_{a c k}\right) \times T_{t x_{-} b}
$$

The total time a node spends during cooperative mechanism transmission at the transmitting side for both $\mathrm{BF}$ and $\mathrm{SM}$ schemes in a cooperative low duty cycle MIMO system is the same as given by Equation (16). The total time a node spends during cooperative mechanism transmission at the receiving side by the SM scheme in a cooperative low duty cycle MIMO system can be given as:

$$
\begin{aligned}
& T_{t x_{-} B r}=r_{s} \times\left(N_{B r}\right) \times T_{t x_{-} b} \\
& T_{t x_{-} c o l}=r_{s} \times(N-1) \times\left(N_{d a t a} \cdot T_{t x_{-} b}+\left(\frac{\sum_{B E=1}^{\max B E} T_{B O}+T_{C C A}}{5}\right)\right)
\end{aligned}
$$

where $N_{B r}$ is the length of broadcast packets at the destination node. $T_{B O}, T_{C C A}$ and $B E$ are the average back-off duration, the clear channel assessment (CCA) analysis duration and the back-off exponent value with all the values derived in detail in (Kohvakka et al., 2006; Kuorilehto et al., 2007). The total time a node spends during data packet transmission for both BF and SM schemes in a cooperative low duty cycle MIMO system is the same as given by Equation (17).

Thus, the total time a node spends during successful transmission for the BF scheme is the same as given in Equation (18) and the total time a node spends during successful transmission for the SM scheme in a cooperative low duty cycle MIMO system can be obtained as:

$$
T_{t x_{-} s_{-} S M}=T_{t x_{-} s_{-} B F}+T_{t x_{-} B r}+T_{t x_{-} c o l}
$$

and the total time a node spends during unsuccessful transmission is the same as in Equation (19) for cooperative BF scheme and is given as:

$$
T_{t x_{-} u_{-} S M}=T_{t x_{-} s_{-} S M}-\left(r_{s} \times N_{a c k} \times T_{t x_{-} b}\right)
$$


for the cooperative SM scheme. The total time a node spends during successful and unsuccessful receptions for both cooperative schemes are the same as in Equations (20) to (24) with an addition for the total time of cooperative mechanism reception at the receiving side by the cooperative SM scheme which is given as:

$$
\begin{aligned}
& T_{r x_{-} B r}=r_{s} \times(N-1) \times N_{B r} \times T_{t x_{-} b} \\
& T_{r x_{-} c o l}=r_{s} \times(N-1) \times N_{d a t a} \times T_{t x_{-} b}
\end{aligned}
$$

The total time a node spends idle for successful communication for both cooperative schemes is given as:

$$
\begin{aligned}
& T_{i d l e_{-} s_{-} B F}=T_{i f s_{-} r t s}+T_{i f s_{-} c t s}+T_{w f c t s}+T_{w f b s d a t a} \\
& T_{\text {idle_s_s }}=T_{\text {idle_s_B } B F}
\end{aligned}
$$

and the idle time for unsuccessful communication is given as:

$$
\begin{aligned}
& T_{\text {idle_u_BF }}=T_{\text {idle_s_BF }}+T_{w f a c k} \\
& T_{\text {idle_u_s }}=T_{\text {idle_s_s }}+T_{w f a c k}
\end{aligned}
$$

where $T_{w f c t s}, T_{w f b s d a t a}$ and $T_{w f a c k}$ are the waiting for the CTS packet period, waiting for the BSDATA packet period and the waiting period for the ACK packet to arrive. The total time a node spends for sleeping for successful communication for both cooperative schemes is given as:

$$
\begin{aligned}
& T_{\text {sleep_s } s_{-} B F}=1-T_{t x_{-} s_{-} B F}-T_{r x_{-} s_{-} B F}-T_{i d l e_{-} s_{-} B F}-T_{\text {listen }}-T_{\text {sensor }} \\
& T_{\text {sleep_s_s }}=1-T_{t x_{-} s_{-} S M}-T_{r x_{-} s_{-} S M}-T_{\text {idle_s_s }}-T_{\text {listen }}-T_{\text {sensor }}
\end{aligned}
$$

and the sleep time for unsuccessful communication is given as:

$$
\begin{aligned}
& T_{\text {sleep_u_BF }}=1-T_{t x_{-} u_{-} B F}-T_{r x_{-} u_{-} B F}-T_{i d l e_{-} u_{-} B F}-T_{\text {listen }} \\
& T_{\text {sleep_u_s }}=1-T_{t x_{-} u_{-} S M}-T_{r x_{-} u_{-} S M}-T_{\text {idle_ }_{-} u_{-} S M}-T_{\text {listen }}
\end{aligned}
$$

Thus, the total energy consumption for successful cooperative low duty cycle MIMO system communication can be obtained as:

$$
\begin{aligned}
& E_{B F_{-} s}=\left(P_{p a}+P_{c t}\right) \cdot T_{t x_{-} s_{-} \text {control }}+\left(P_{p a B s}+P_{c t}\right) \cdot T_{t x_{-} B s d a t a}+ \\
& \left(P_{p a B F}+P_{c t}\right) \cdot T_{t x_{-} \text {data }}+\left(P_{r}+P_{c r}\right) \cdot T_{r x_{-} s_{-} \text {control }}+\left(P_{r B s}+P_{c r}\right) \cdot T_{r x_{-} \text {Bsdata }}+ \\
& \left(P_{r B F}+P_{c r}\right) \cdot T_{r x_{-} \text {data }}+P_{\text {idle }} \cdot T_{\text {idle_s_B } B F}+P_{\text {sleep }} \cdot T_{\text {sleep_s_B } s_{-} B}+E_{\text {listen }}
\end{aligned}
$$


and

$$
\begin{aligned}
& E_{S M_{-} s}=\left(P_{p a}+P_{c t}\right) \cdot T_{t x_{-} s_{-} \text {control }}+\left(P_{p a B s}+P_{c t}\right) \cdot T_{t x_{-} B s d a t a}+ \\
& \left(P_{p a B F}+P_{c t}\right) \cdot T_{t x_{-} \text {data }}+\left(P_{p a B r}+P_{c t}\right) \cdot T_{t x_{-} B r}+\left(P_{p a B r}+P_{c t}\right) \cdot T_{t x_{-} c o l}+ \\
& \left(P_{r}+P_{c r}\right) \cdot T_{r x_{-} s_{-} c o n t r o l}+\left(P_{r B s_{1}}+P_{c r}\right) \cdot T_{r x_{-} B s d a t a}+\left(P_{r S M}+P_{c r}\right) \cdot T_{r x_{-} \text {data }}+ \\
& P_{\text {idle }} \cdot T_{\text {idle_s_s } s_{-} S}+P_{\text {sleep }} \cdot T_{\text {sleep_s_s } s_{-} S M}+E_{\text {listen }}
\end{aligned}
$$

and the total energy consumption for unsuccessful cooperative low duty cycle MIMO system communication can be obtained as:

$$
\begin{aligned}
& E_{B F_{-} u}=\left(P_{p a}+P_{c t}\right) \cdot T_{t x_{-} u_{-} \text {control }}+\left(P_{p a B s}+P_{c t}\right) \cdot T_{t x_{-} B s d a t a}+ \\
& \left(P_{p a B F}+P_{c t}\right) \cdot T_{t x_{-} \text {data }}+\left(P_{r}+P_{c r}\right) \cdot T_{r x_{-} u_{-} \text {control }}+\left(P_{r B s}+P_{c r}\right) \cdot T_{r x_{-} B s d a t a}+ \\
& \left(P_{r B F}+P_{c r}\right) \cdot T_{r x_{-} \text {data }}+P_{\text {idle }} \cdot T_{\text {idle_u_B }_{-} B F}+P_{\text {sleep }} \cdot T_{\text {sleep_u_BF }}+E_{\text {listen }}
\end{aligned}
$$

and

$$
\begin{aligned}
& E_{S M_{-} u}=\left(P_{p a}+P_{c t}\right) \cdot T_{t x_{-} u_{-} \text {control }}+\left(P_{p a B s}+P_{c t}\right) \cdot T_{t x_{-} B s d a t a}+ \\
& \left(P_{p a B F}+P_{c t}\right) \cdot T_{t x_{-} \text {data }}+\left(P_{p a B r}+P_{c t}\right) \cdot T_{t x_{-} B r}+\left(P_{p a B r}+P_{c t}\right) \cdot T_{t x_{-} c o l}+ \\
& \left(P_{r}+P_{c r}\right) \cdot T_{r x_{-} u_{-} \text {control }}+\left(P_{r B s}+P_{c r}\right) \cdot T_{r x_{-} B s d a t a}+\left(P_{r S M}+P_{c r}\right) \cdot T_{r x_{-} \text {data }}+ \\
& P_{\text {idle }} \cdot T_{\text {idle_u_sM }}+P_{\text {sleep }} \cdot T_{\text {sleep_u_s } u_{-}}+E_{\text {listen }}
\end{aligned}
$$

Therefore, the total energy consumption for the cooperative low duty cycle MIMO system can be modelled as a function of the retransmission rate:

$$
\begin{gathered}
E_{B F}=\left(\frac{P_{p B F}}{1-P_{p B F}}\right) E_{B F_{-} u}+E_{B F_{-} s}+E_{\text {sensor }} \\
E_{S M}=\left(\frac{P_{p S M}}{1-P_{p S M}}\right) E_{S M_{-} u}+E_{S_{M_{-} s}}+E_{\text {sensor }}
\end{gathered}
$$

where $P_{p B F}$ and $P_{p S M}$ are the packet error probability of the cooperative BF and SM systems respectively which can be obtained from (Ahmad et al., 2008a).

\section{Packet Latency Performance Model}

As we noted, each packet transmission in cooperative transmission requires more steps which introduces more overhead. These steps may increase packet delays. However, the 
reduction of PER as the diversity gain increases from the cooperative MIMO exploitation can reduce the retransmissions rates which in turn can reduce packet latency. Previous work in (Ahmad et al., 2008a) models packet latency performance for the non-cooperative SISO system. Comparison is then made with the models developed for the cooperative MIMO systems as shown in (Ahmad et al., 2008b). In addition to the delay incurred as calculated and analyzed in (Ahmad et al., 2008a \& 2008b) for CMACON with both BF and SM cooperative schemes, the cyclic RTS-CTS transmission scheme periods which are calculated in Equation (1) are included. Also, the IFS periods for both RTS and CTS packet transmissions as calculated in Equation (2) are included.

\section{Performance Analysis and Discussion}

All the important parameters for energy consumption modelling are listed in (Mainwaring et al., 2002; Cui et al., 2004; Polastre et al., 2004; Yang et al., 2007) with the times taken to transmit and receive 1 bit, $T_{r x_{-} b}$ and $T_{t x_{-} b}$ fixed at $4 \mu$ s corresponding to the bit rate of the system. The values of the system parameters used in Figures 14 and 15 for latency analysis are as follows: $T_{r t s}=0.52 \mathrm{~ms}, T_{c t s}=0.44 \mathrm{~ms}, T_{a c k}=0.432 \mathrm{~ms}, T_{B s}=4.528 \mathrm{~ms}, T_{B r}=0.432 \mathrm{~ms}$, $T_{\text {data }}=4.096 \mathrm{~ms}, T_{\text {col }}=32.8 \mathrm{~ms}$, and $T_{\text {wfack }}$ for $S M$ scheme $=70 \mathrm{~ms}$ (Yang et al., 2007) and $T_{\text {wfack }}$ for BF scheme $=0.864 \mathrm{~ms}$ (Kohvakka et al., 2006).

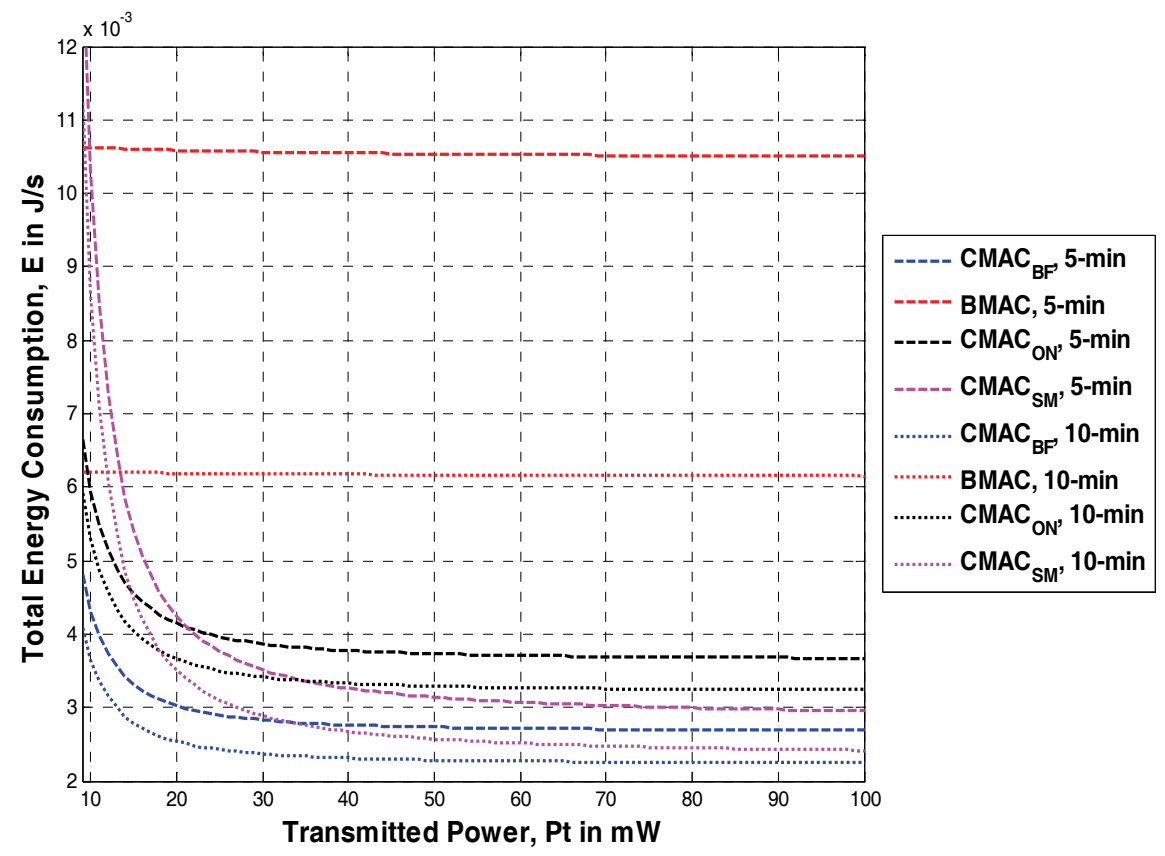

Fig. 5. Total energy consumption vs. transmission power of various MAC protocols with $M$ $=2$ and $N=1$ (Cooperative $\mathrm{BF}$ ) and $M=N=2$ (Cooperative SM) for 5-min and 10-min sample periods 


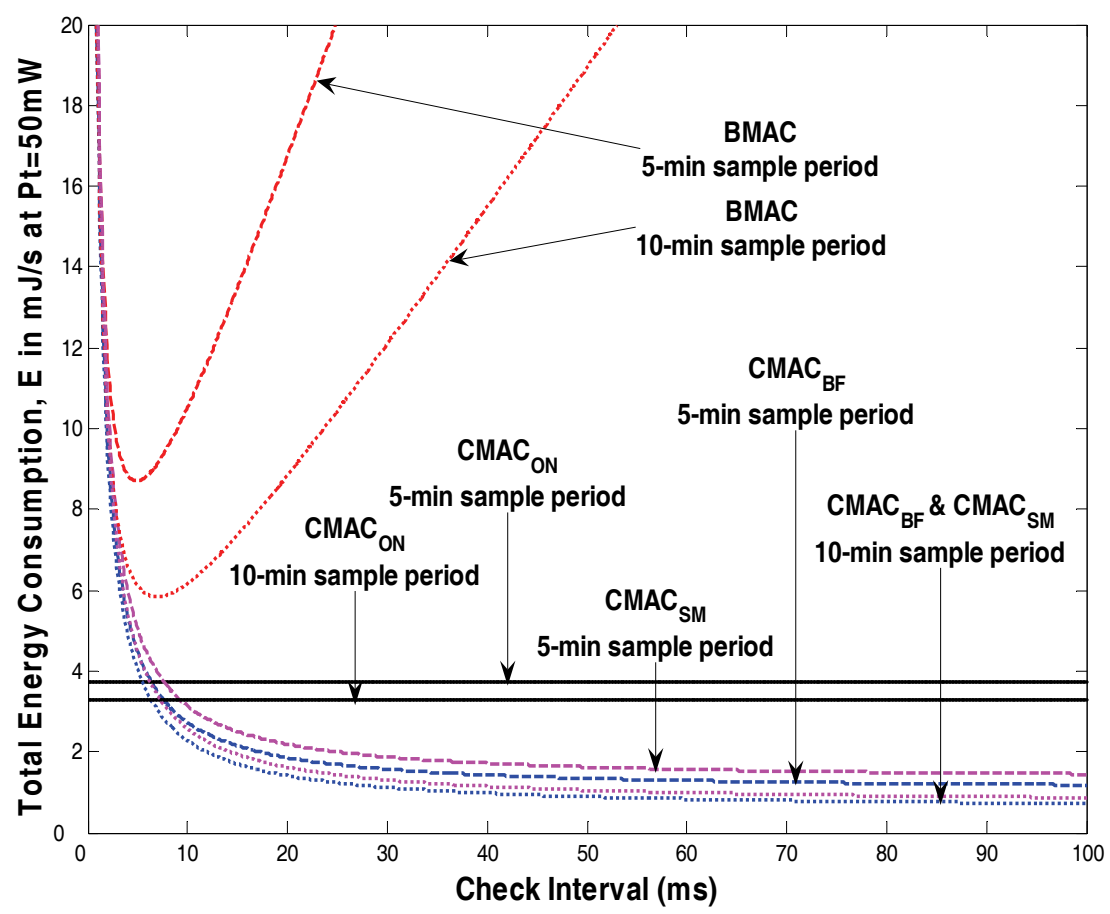

Fig. 6. Total energy consumption vs. check interval of various MAC protocols with $M=2$ and $N=1$ (Cooperative BF) and $M=N=2$ (Cooperative SM) for 5-min and 10-min sample periods

We can see in Figure 5 that both CMAC and $\mathrm{CMAC}_{\mathrm{ON}}$ outperform B-MAC and that the $\mathrm{CMAC}_{\mathrm{BF}}$ is more energy efficient than $\mathrm{CMAC}_{\mathrm{SM}}$ with two transmitting nodes for all the sampling periods. If we let the sampling period be long enough, the performance difference between CMAC and B-MAC should be reduced at the same check interval. Thus, we can deduce that CMAC is more energy efficient than B-MAC at shorter sampling periods which makes CMAC more practical for applications with frequent sampling periods.

As shown in Figure 6, B-MAC has the optimal check interval at $5 \mathrm{~ms}$ for the 5 minutes sampling period. We can expect that the optimal check interval gets higher when the sampling period gets higher. As measured at 10 minutes sampling period, the optimal check interval is $7 \mathrm{~ms}$ with $2 \mathrm{~ms}$ increase. The same observation is applied for CMAC as shown in Figure 7. Furthermore from Figure 6, we can observe that below $3 \mathrm{~ms}$, both B-MAC and CMAC suffer higher transient energy which puts the lower bound or lower constraint on the operating check interval. Clearly, above $7 \mathrm{~ms}, \mathrm{CMAC}$ outperforms both $\mathrm{CMAC}_{\mathrm{ON}}$ and BMAC. B-MAC may suffer from higher transmission power due to a longer preamble packet as the check interval gets higher. Interestingly, $\mathrm{CMAC}_{\mathrm{SM}}$ has the same optimal check interval with $\mathrm{CMAC}_{\mathrm{BF}}$ for various sampling periods as shown in Figure 7. 


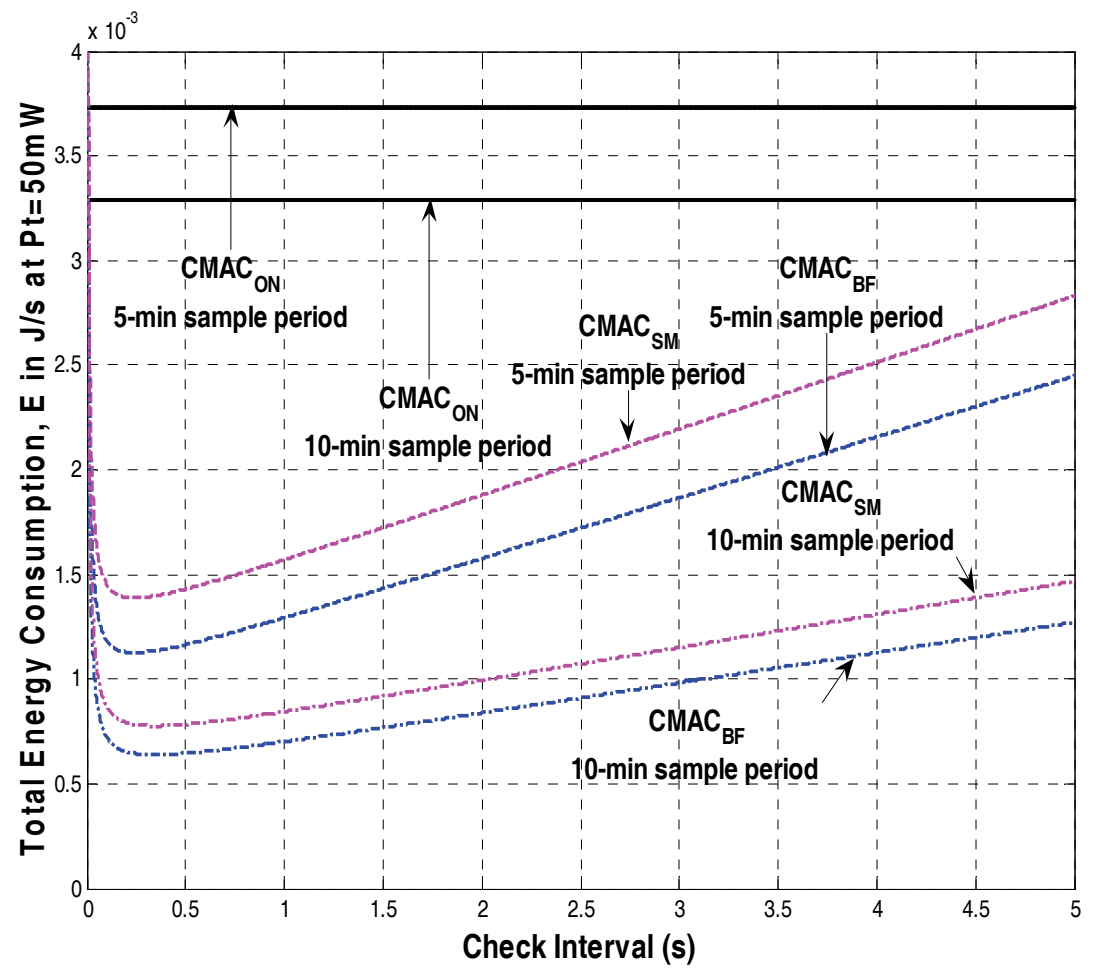

Fig. 7. Total energy consumption vs. check interval of CMAC protocols when $M=2$ and $N=$ 1 (Cooperative $\mathrm{BF}$ ) and $M=N=2$ (Cooperative SM) for 5-min and 10-min sample periods

Figure 8 shows the impact of $M$ on the energy consumption of CMAC and CMACON. We can observe that the increase of energy consumption is small as $M$ increases even when we increase $M$ from 2 to 10 nodes. As long as the nodes are operating within an optimal range during cooperative communication (Nguyen et al., 2007), the small circuit energy can be tolerated in a cooperative low duty cycle MIMO system. The impact of $N$ is shown in Figure 9. As we observed earlier, increasing $M$ does not have a significant impact on the total energy consumption for both schemes. Interestingly, we also observe that $N$ does not have a significant impact on the total energy consumption. Therefore, as long as we can tolerate a little increase of circuit energy by increasing the number of $M$ and $N$, then we can choose to use either the BF or SM scheme in a cooperative low duty cycle MIMO system. However, the optimal choice is still to use $\mathrm{CMAC}_{\mathrm{BF}}$ and to set $M=2$ and this result agrees with the previous results in (Ahmad et al., 2008a). On the other hand, when we consider high-speed WSNs, obviously $\mathrm{CMAC}_{\mathrm{SM}}$ is the optimal choice. 


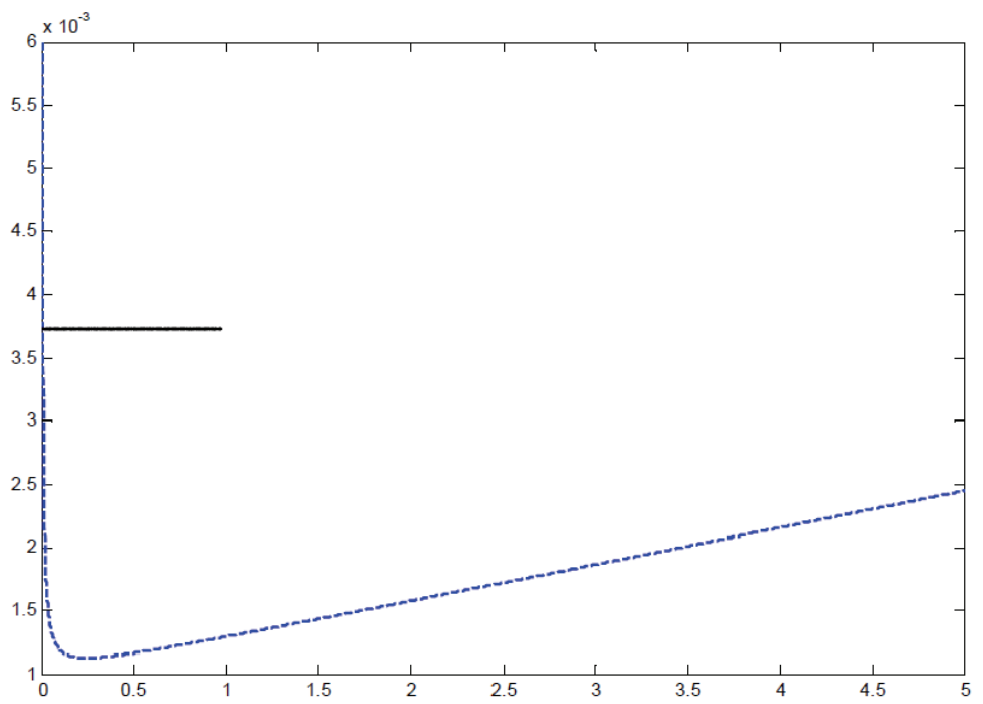

Fig. 8. Total energy consumption vs. check interval of CMAC protocols for various $M$ with $N=1$ (Cooperative BF) and $N=2$ (Cooperative SM)

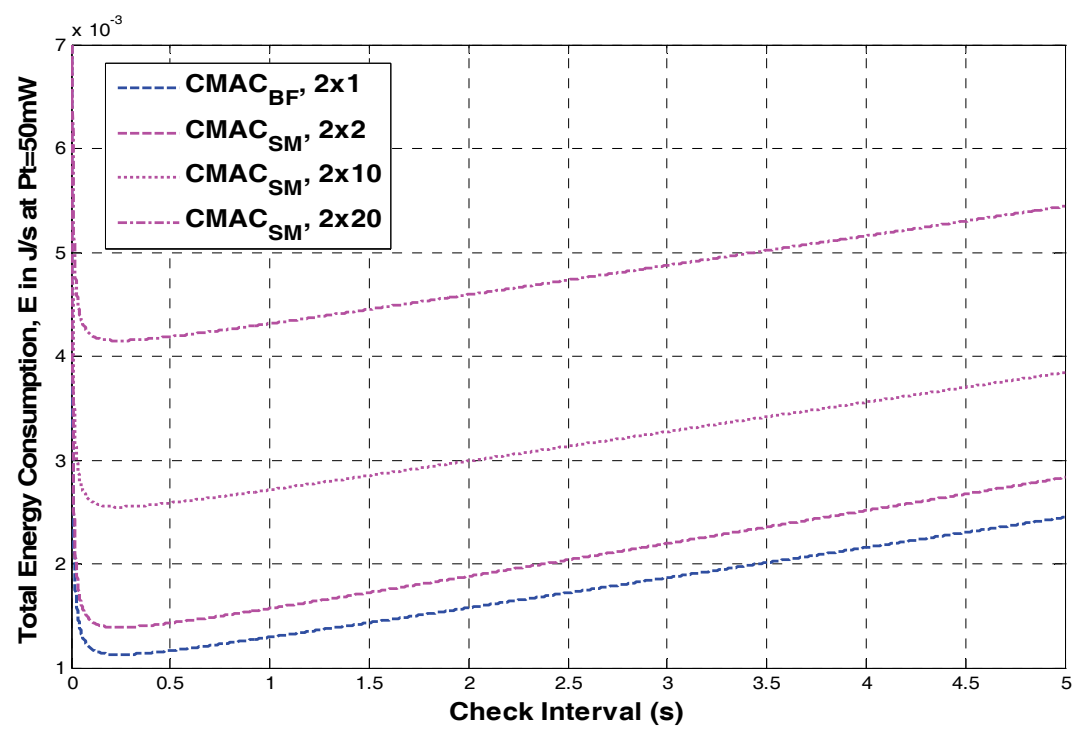

Fig. 9. Total energy consumption vs. check interval of CMAC protocols for various $N$ (Cooperative $\mathrm{SM}$ ) with fixed $M=2$ for all cooperative schemes 


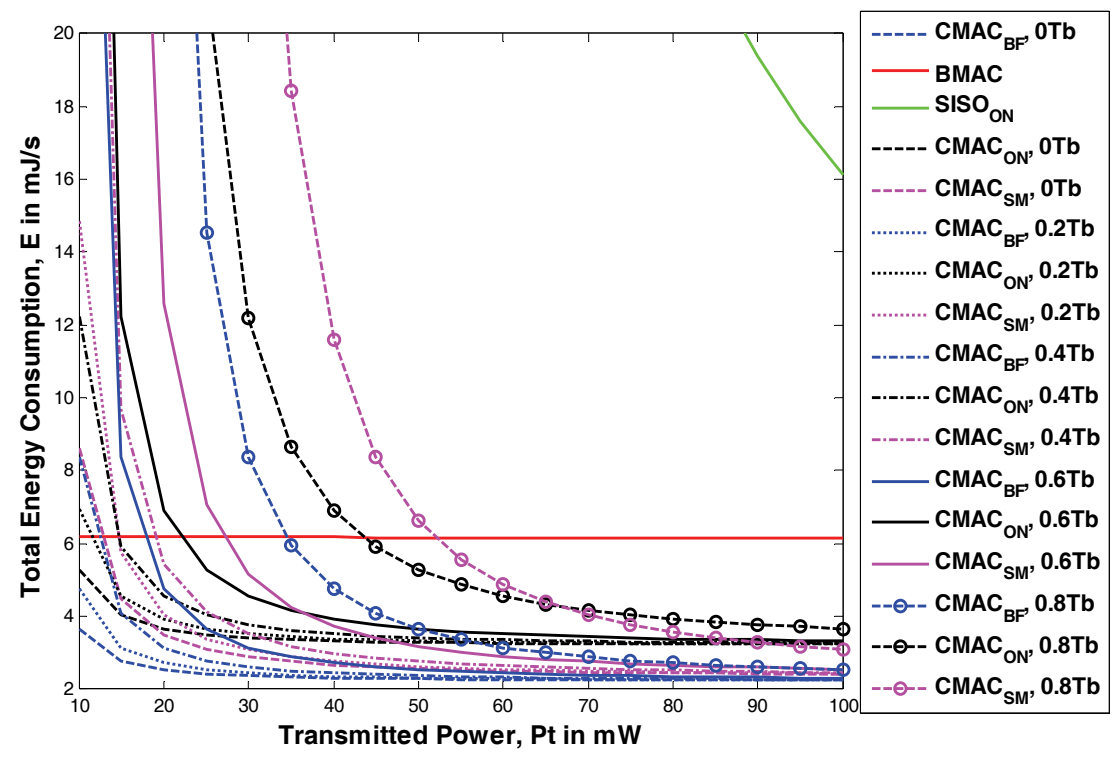

Fig. 10. Total energy consumption vs. transmission power for various imperfect synchronization cooperative schemes with $M=2$ and $N=1$ (Cooperative BF) and $M=N=2$ (Cooperative SM)

Figure 10 shows that $\mathrm{CMAC}_{\mathrm{BF}}$ outperforms the other schemes below $0.8 T_{b}$ at common transmission power above $40 \mathrm{~mW}$. Figure 11 shows the CMAC $C_{S M}$ suffers the timing error effect at above $0.9 T_{b}$ where SISO $\mathrm{ON}_{\text {outperforms }} \mathrm{CMAC}_{\mathrm{BF}}$. Also we observe that B-MAC outperforms both $\mathrm{CMAC}_{\mathrm{BF}}$ and $\mathrm{CMAC}_{\mathrm{ON}}$ utilizing the $\mathrm{BF}$ scheme with $0.9 \mathrm{~T}_{b}$ at a lower check interval below $200 \mathrm{~ms}$. A closer look at all the cooperative MAC schemes is shown in Figure 12 where the jitter difference is varied from $0 T_{b}$ to $0.8 T_{b} . \mathrm{CMAC}_{\mathrm{BF}}$ experiences $1.3 \mathrm{~mJ} / \mathrm{s}$ increases between $0 T_{b}$ and $0.8 T_{b}$. The increase is still small when we compare it to $\mathrm{CMAC}_{\mathrm{SM}}$ and $\mathrm{CMAC}_{\mathrm{ON}}$ utilising the $\mathrm{BF}$ scheme with $4.6 \mathrm{~mJ} / \mathrm{s}$ and $3.5 \mathrm{~mJ} / \mathrm{s}$ increases, respectively.

The impact of the number of cooperative receiving nodes, $N$, in the cooperative SM scheme is shown in Figure 13. We can reduce the energy cost from $4.6 \mathrm{~mJ} / \mathrm{s}$ increase to $0.2 \mathrm{~mJ} / \mathrm{s}$ increase when $N=6$. As $N$ gets higher, the circuit energy gets higher and thus the total energy consumption also gets higher. However, we can tolerate the small circuit energy at higher jitter differences as shown since $\mathrm{CMAC}_{\mathrm{ON}}$ utilising the $\mathrm{BF}$ scheme with $N=20$ at $0.8 T_{b}$ has lower energy than $\mathrm{CMAC}_{\mathrm{ON}}$ utilizing the $\mathrm{BF}$ scheme with $N=2$ at $0.8 \mathrm{~T}_{b}$. From all the observations, we suggest that $\mathrm{CMAC}_{\mathrm{BF}}$ is the optimal choice below $0.9 T_{b}$ jitter difference. As shown in Figures 14 and 15, B-MAC enjoys lower packet latency and outperforms the other schemes even when the diversity gain of the cooperative SM scheme is increased. $\mathrm{CMAC}_{\mathrm{ON}}$ utilising the $\mathrm{BF}$ scheme outperforms B-MAC when the transmission power is higher than $50 \mathrm{~mW}$ below $0.4 T_{b}$. CMAC $\mathrm{CF}_{\mathrm{BF}}$ with $0 T_{b}$ suffers a slightly higher delay compared to B-MAC when the transmission power is $50 \mathrm{~mW}$. In order to maintain lower latency, as low as $50 \mathrm{~ms}, \mathrm{CMAC}_{\mathrm{BF}}$ must operate below $0.6 T_{b}$ jitter difference. 


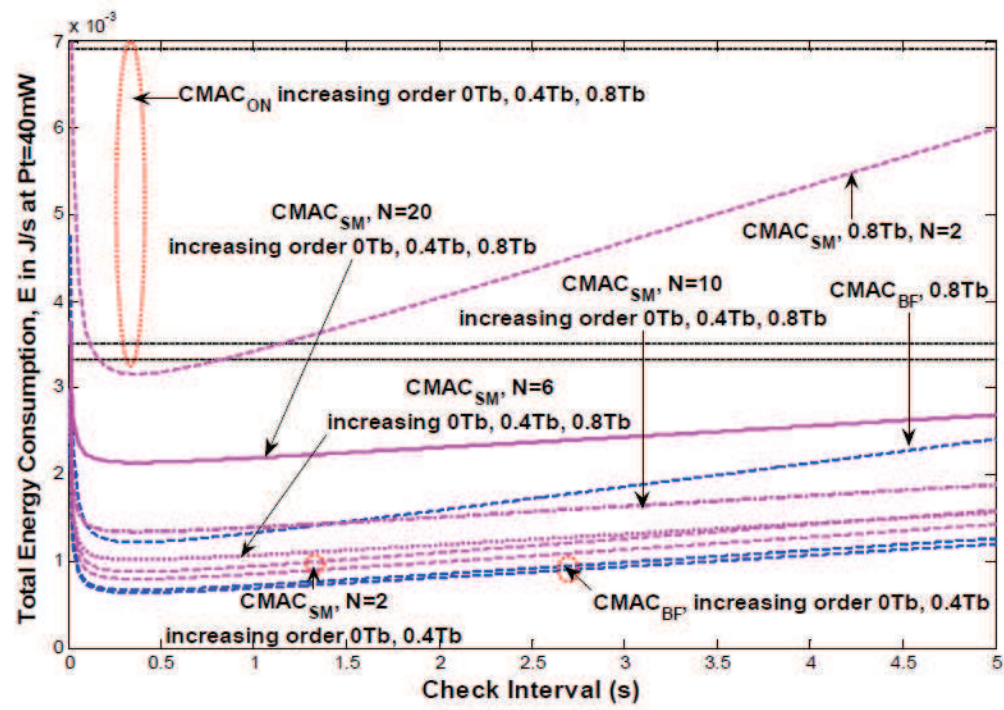

Fig. 11. Total energy consumption vs. check interval for various imperfect synchronisation cooperative schemes with $M=2$ and $N=1$ (Cooperative $\mathrm{BF}$ ) and $M=N=2$ (Cooperative $\mathrm{SM})$ at clock jitter $=0.9 \mathrm{~T}_{b}$

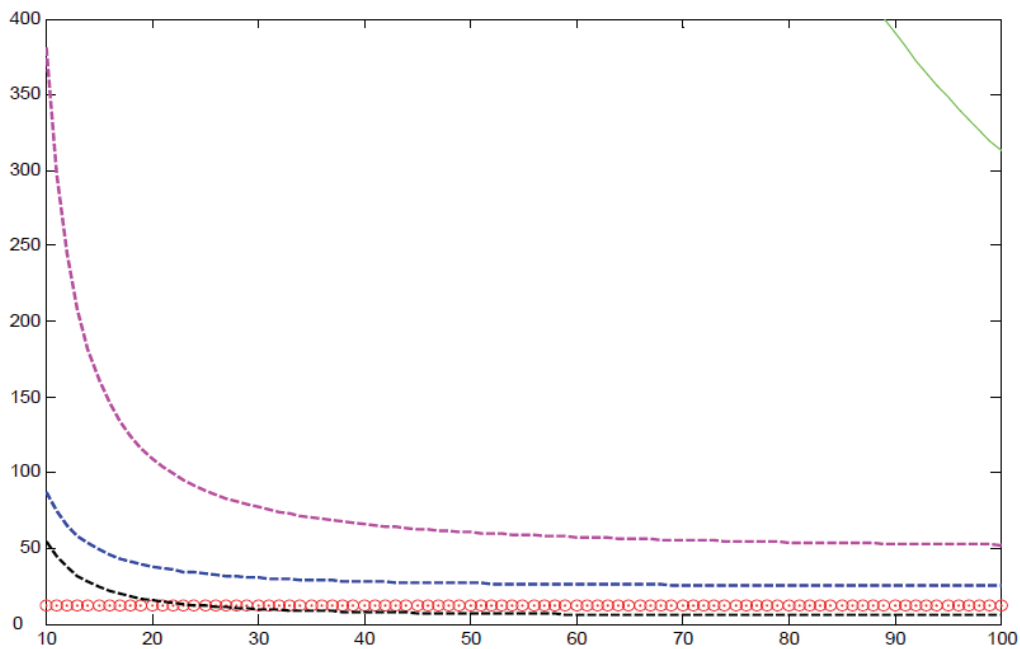

Fig. 12. Total energy consumption vs. check interval for various imperfect synchronisation schemes with $M=2$ and $N=1$ (Cooperative BF) and $M=N=2$ (Cooperative SM) with clock jitter $\leq 0.8 T_{b}$ 


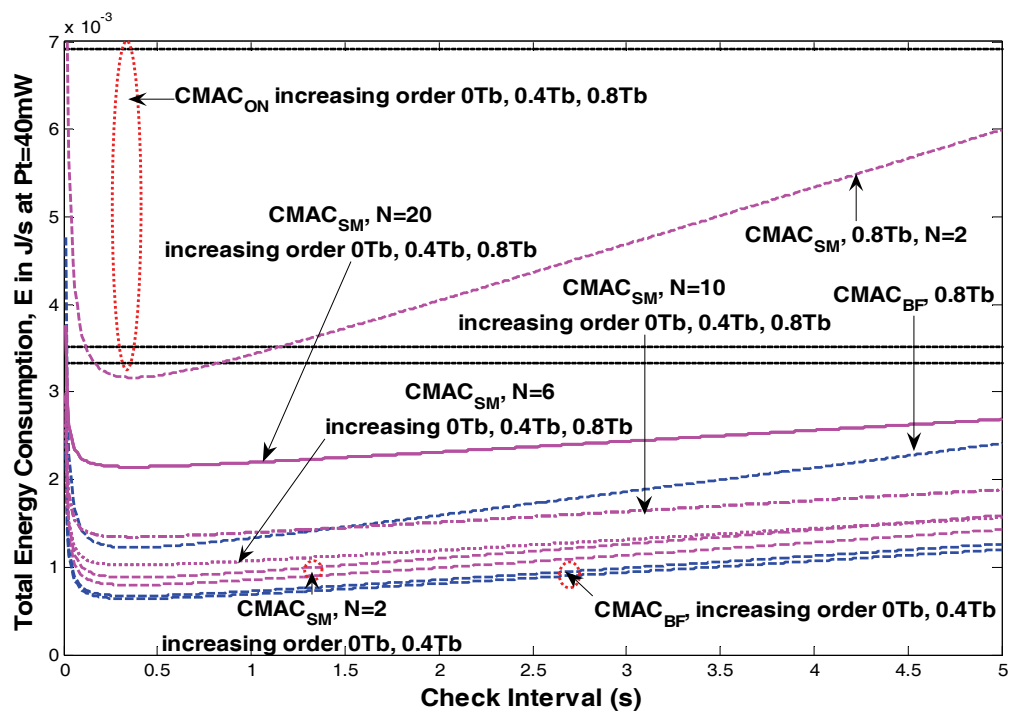

Fig. 13. Total energy consumption vs. check interval for various imperfect synchronization schemes with $M=2$ and $N=1$ (Cooperative $\mathrm{BF)}$ ) and with $M=2$ and various $N=2,6,10$, and 20 (Cooperative SM) with clock jitter $\leq 0.8 T_{b}$

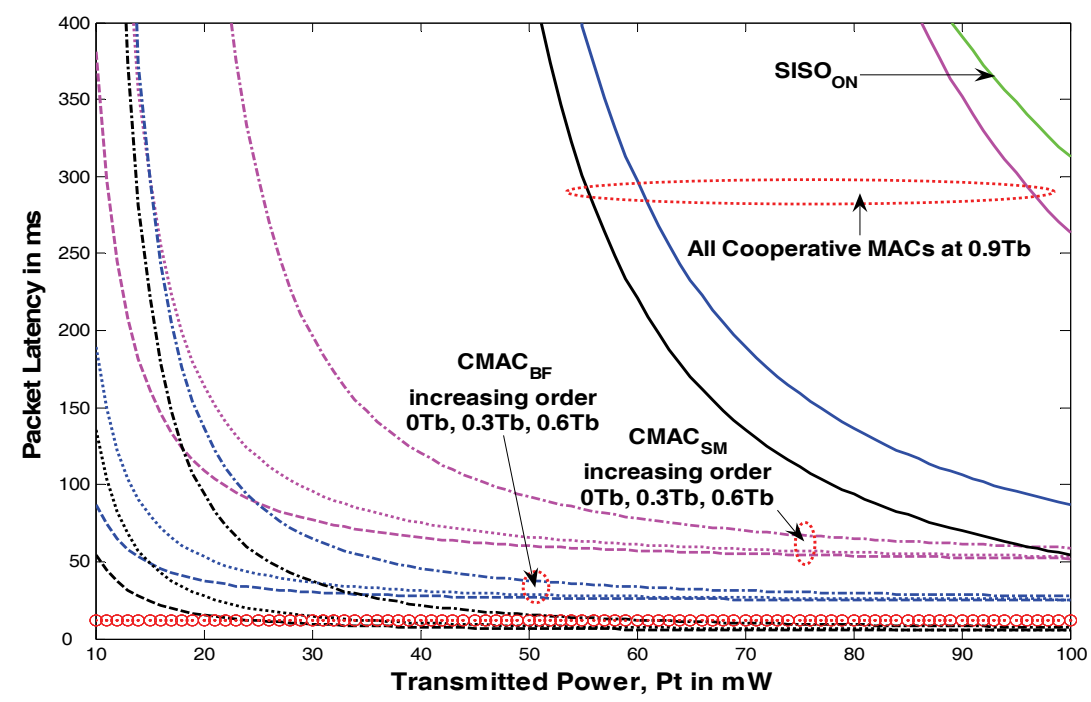

Fig. 14. Packet latency vs. transmission power of various imperfect synchronization schemes with $M=2$ and $N=1$ (Cooperative $\mathrm{BF}$ ) and $M=N=2$ (Cooperative SM) for $0 T_{b}, 0.3 T_{b}, 0.6 T_{b}$ and $0.9 T_{b}$ 


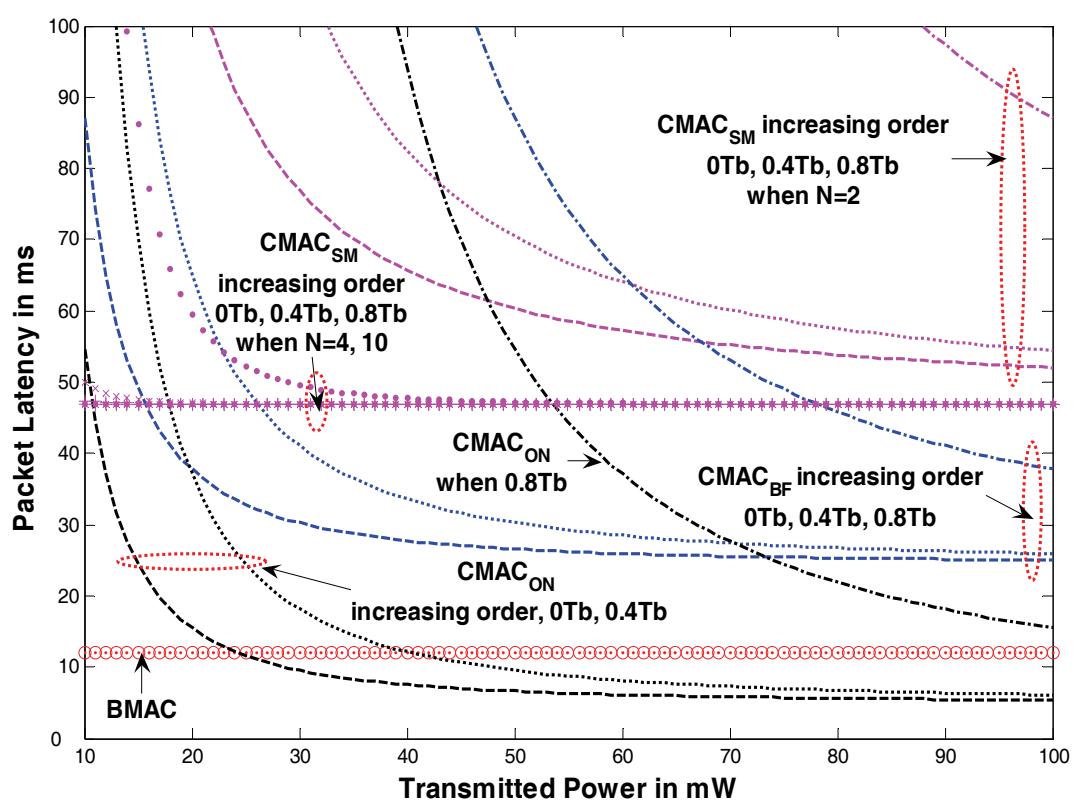

Fig. 15. Packet latency vs. transmission power of various imperfect synchronization schemes with $M=2$ and $N=1$ (Cooperative $\mathrm{BF}$ ) and with $M=2$ and various $N=2,4$, and 10 (Cooperative $\mathrm{SM}$ ) for $0 T_{b}, 0.4 T_{b}$ and $0.8 T_{b}$

\section{Conclusions}

In order to address the idle listening and overhearing problems in a system with the $\mathrm{CMAC}_{\mathrm{ON}}$ protocol, we have proposed a new Cooperative low duty cycle MAC protocol (CMAC) for two cooperative MIMO schemes: optimal Beamforming $\left(\mathrm{CMAC}_{\mathrm{BF}}\right)$ and Spatial Multiplexing $\left(\mathrm{CMAC}_{\mathrm{SM}}\right)$. We have developed analytical models to evaluate total energy consumption and packet latency for both schemes. We have considered both synchronous and asynchronous scenarios. We have taken into consideration all the related energy costs (transmission, reception, idle listening, establishing cooperative mechanism, sleep, etc.) in the system performance modeling. We have applied the models for periodic monitoring applications.

We conclude that the new cooperative low duty cycle MAC with the optimal Beamforming scheme $\left(\mathrm{CMAC}_{\mathrm{BF}}\right)$ outperforms the other cooperative and SISO schemes in terms of total energy consumption with the number of cooperating nodes set to $M=2$. In order to achieve both lower energy and lower latency, $\mathrm{CMAC}_{\mathrm{BF}}$ must operate at $M=2$ and with the clock jitter difference below $0.6 \mathrm{~Tb}$. These results can be used to assist with the design of CMAC for multi-hop communication. Moreover, the trade-off relationship between energy efficient operation and latency can be utilized to find the optimal number of hops and the optimal number of cooperating nodes that should be involved in the transmission. 


\section{References}

Ahmad, M.R.; Dutkiewicz, E. \& Huang, X. (2008a). Performance Analysis of Cooperative MIMO Transmission Schemes in WSN, Proceedings of IEEE International Symposium on Personal, Indoor and Mobile Radio Communications (PIMRC), Cannes, France, 15-18 September 2008.

Ahmad, M.R.; Dutkiewicz, E. \& Huang, X. (2008b). Performance Evaluation of MAC Protocols for Cooperative MIMO Transmissions in Sensor Networks, Proceedings of ACM International Workshop on Performance Evaluation of Wireless Ad-hoc, Sensor, and Ubiquitous Networks, Vancouver, Canada, 2008.

Buettner, M.; Yee, G.; Anderson, E. \& Han, R. (2006). X-MAC: A Short Preamble MAC Protocol for Duty-Cycled Wireless Sensor Networks, Proceedings of ACM Conference on Embedded Networked Sensor Systems (SENSYS), Baltimore, Maryland, USA, 2006.

Cui, S.; Goldsmith, A.J. \& Bahai, A. (2004). Energy-efficiency of MIMO and Cooperative MIMO Techniques in Sensor Networks. IEEE Journal on Selected Areas in Communications, Vol. 22, No. 6, pp. 1089-1098.

Jagannathan, S.; Aghajan, H. \& Goldsmith, A.J. (2004). The Effect of Time Synchronization Errors on the Performance of Cooperative MISO Systems, Proceedings of IEEE Global Telecommunications Conference (GLOBECOM), Dallas, Texas, USA, 2004.

Kohvakka, M.; Kuorilehto, M.; Hannikainen, M. \& Hamalainen, T.D. (2006). Performance Analysis of IEEE 802.15.4 and Zigbee for large-Scale Wireless Sensor Network Applications, Proceedings of ACM International Workshop on Performance Evaluation of Wireless Ad-hoc, Sensor, and Ubiquitous Networks, Malaga, Spain, 2006.

Kuorilehto, M.; Kohvakka, M.; Suhonen, J.; Hamalainen, P.; Hannikainen, M. \& Hamalainen, T.D. (2007). MAC Protocols, In: Ultra-Low Energy Wireless Sensor Networks in Practice, pp. 73-88, John Wiley, 978-0-470-05786-5, West Sussex, England.

Mainwaring, A.; Polastre, J.; Szewczyk, R.; Culler, D. \& Anderson, J. (2002). Wireless Sensor Networks for Habitat Monitoring, Proceedings of ACM International Workshop on Wireless Sensor Networks and Applications, 2002.

Nguyen, T.-D.; Berder, O. \& Sentieys, O. (2007). Cooperative MIMO Schemes Optimal Selection for Wireless Sensor Networks, Proceedings of IEEE Vehicular Technology Conference (VTC), Baltimore, Maryland, USA, 2007.

Polastre, J.; Hill, J. \& Culler, D. (2004). Versatile Low Power Media Access for Wireless Sensor Networks, Proceedings of ACM Conference on Embedded Networked Sensor Systems (SENSYS), pp. 95-107, Baltimore, Maryland, USA, November 2004.

Wong, K.J. \& Arvind, D. (2006). Low Power Decentralized MAC Protocols for Low Data Rate Transmissions in Specknets, Proceedings of ACM International Workshop on Multihop Ad-hoc Networks: From Theory to Reality, Florence, Italy, 2006.

Yang, H.; Shen, H.-Y. \& Sikdar, B. (2007). A MAC Protocol for Cooperative MIMO Transmissions in Sensor Networks, Proceedings of IEEE Global Telecommunications Conference (GLOBECOM), pp. 636-640, ISBN 1-4244-1042-8, Washington DC, USA, 26-30 November 2007. 


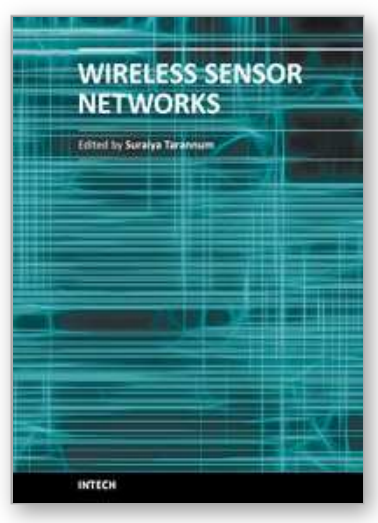

\section{Wireless Sensor Networks \\ Edited by}

ISBN 978-953-307-325-5

Hard cover, 342 pages

Publisher InTech

Published online 29, June, 2011

Published in print edition June, 2011

\section{How to reference}

In order to correctly reference this scholarly work, feel free to copy and paste the following:

Mohd Riduan Ahmad, Eryk Dutkiewicz and Xiaojing Huang (2011). Energy Efficient Cooperative MAC Protocols in Wireless Sensor Networks, Wireless Sensor Networks, (Ed.), ISBN: 978-953-307-325-5, InTech, Available from: http://www.intechopen.com/books/wireless-sensor-networks/energy-efficient-cooperative-macprotocols-in-wireless-sensor-networks

\section{INTECH}

open science | open minds

\section{InTech Europe}

University Campus STeP Ri

Slavka Krautzeka 83/A

51000 Rijeka, Croatia

Phone: +385 (51) 770447

Fax: +385 (51) 686166

www.intechopen.com

\section{InTech China}

Unit 405, Office Block, Hotel Equatorial Shanghai

No.65, Yan An Road (West), Shanghai, 200040, China

中国上海市延安西路65号上海国际贵都大饭店办公楼405单元

Phone: +86-21-62489820

Fax: +86-21-62489821 
(C) 2011 The Author(s). Licensee IntechOpen. This chapter is distributed under the terms of the Creative Commons Attribution-NonCommercialShareAlike-3.0 License, which permits use, distribution and reproduction for non-commercial purposes, provided the original is properly cited and derivative works building on this content are distributed under the same license. 